\title{
Características psicométricas do Questionário de Temperamento de Aproximação e Evitação em trabalhadores
}

\author{
Alexandra de Oliveira Rodrigues-Marçulo ${ }^{1}{ }^{1}$ \\ Maria Cristina Ferreira (10 1 \\ Michelle Morelo Pereira ${ }^{1}{ }^{1}$ \\ ${ }^{1}$ Universidade Salgado de Oliveira. Niterói, RJ, Brasil.
}

\begin{abstract}
Resumo
O temperamento de aproximação ou abordagem caracteriza-se como uma sensibilidade neurobiológica geral a estímulos positivos desejáveis, enquanto o temperamento de evitação ou evasão é instigado por eventos negativos indesejáveis. O presente trabalho reúne evidências iniciais de validade de estrutura interna e de relações com variáveis externas do Questionário de Temperamento de Aproximação-Evitação (ATQ) em trabalhadores brasileiros. Participaram do estudo 453 trabalhadores de ambos os sexos (79,2\% do sexo feminino). As análises fatoriais confirmatórias indicaram que o modelo ajustado de dois fatores não correlacionados foi o que obteve os melhores índices de ajuste. Na relação com variáveis externas, as dimensões de aproximação e evitação apresentaram, respectivamente, correlações positivas baixas e correlações negativas baixas a moderadas, com o engajamento no trabalho, a adaptabilidade à carreira e o desempenho de papéis no trabalho. As evidências iniciais de validade obtidas recomendam o uso do ATQ em pesquisas futuras realizadas no contexto organizacional brasileiro.
\end{abstract}

Palavras-chave: traços de personalidade; trabalho; validade estatística; psicometria.

\section{Psychometric characteristics of the Approach-Avoidance Temperament Questionnaire in workers}

\begin{abstract}
Approach temperament is characterized as a general neurobiological sensitivity to desirable positive stimuli, while the avoidance temperament is instigated by undesirable negative events. The study gathered initial evidence of validity of internal structure and relations with external variables of the Approach-Avoidance Temperament Questionnaire (ATQ) in Brazilian workers. In total, 453 workers of both gender (79.2\% female) participated in the study. Confirmatory factor analyses indicated that the adjusted model of two uncorrelated factors was the one that obtained the best adjustment indices. In relation to external variables, the dimensions of approach and avoidance presented, respectively, low positive correlations and low to moderate negative correlations, with work engagement, career adaptability and role performance at work. The initial evidence of validity obtained recommends the use of the ATQ in future research carried out in the Brazilian organizational context.
\end{abstract}

Keywords: personality traits; job, statistical validity, psychometry.

\section{Características psicométricas del Cuestionario de Temperamento de Aproximación y Evitación en trabajadores}

Resumen

El temperamento de aproximación o enfoque se caracteriza como una sensibilidad neurobiológica general a estímulos positivos deseables, mientras que el temperamento de evitación o evasión es instigado por eventos negativos indeseables. El estudio reunió evidencias iniciales de validez de estructura interna y de relaciones con variables externas del Cuestionario de Temperamento de Aproximación-Evitación (ATQ) en trabajadores brasileños. Participaron del estudio 453 trabajadores de ambos sexos (79,2\% del sexo femenino). Las análisis factoriales confirmatorios indicaron que el modelo ajustado de dos factores no correlacionados fue el que obtuvo los mejores índices de ajuste. En la relación con variables externas, las dimensiones de aproximación y evitación presentaron, respectivamente, correlaciones positivas bajas y correlaciones negativas bajas a moderadas, con el compromiso en el trabajo, la adaptabilidad a la carrera y el desempeño de papeles en el trabajo. Las evidencias iniciales de validez obtenidas recomiendan el uso del ATQ en investigaciones futuras realizadas en el contexto organizacional brasileño.

Palabras clave: rasgos de personalidad; trabajo; validez estadística; psicometría.

Os comportamentos de aproximação e evitação têm uma longa história, com o interesse por essa temática 
remontando aos antigos filósofos gregos, como, por exemplo, Demócrito (460-370 a.C.) e Aristippus (435356 a.C.), para os quais um hedonismo ético, voltado à busca do prazer e à evitação da dor, era central para o comportamento humano. Mais tarde, um dos primeiros pensadores a discutir diretamente o hedonismo psicológico como busca do prazer e evitação da dor foi o filósofo britânico Jeremy Bentham (1748-1832). De acordo com ele, tal princípio descrevia fielmente o modo pelo qual as pessoas tendiam a se comportar (Elliot, 2008).

A decisão de se aproximar ou evitar caracterizase como uma decisão adaptativa fundamental aos organismos ao longo da história evolutiva, visto que a capacidade de discriminar entre os estímulos agradáveis ou gratificantes e perigosos, que permitem a escolha entre a aproximação ou a evitação, revelase crucial para a sobrevivência humana (Tooby \& Cosmides, 1990). Por essa razão, durante os últimos 30 anos, vários teóricos têm se dedicado aos estudos das bases neuroanatômicas subjacentes à motivação para os comportamentos de aproximação e evitação.

Embora diferindo em uma variedade de formas, esses modelos teóricos têm em comum a proposição de que existem substratos neuroanatômicos identificáveis que se localizam no cérebro e se encontram subjacentes aos processos motivacionais de aproximação e evitação (Elliot, 2008). Nesse sentido, os comportamentos de aproximação estão associados à ativação do córtex préfrontal esquerdo, enquanto a retirada ou evasão está relacionada ao córtex pré-frontal direito, sendo que tais substratos são relativamente independentes e geridos por estruturas diferentes do sistema nervoso, conforme evidenciado em investigações neurofisiológicas (Elliot, 2008).

Na Psicologia, muitos dos estudiosos da motivação humana também incorporaram em suas conceituações e teorizações a distinção entre os temperamentos de aproximação (ou abordagem) e evitação (ou evasão). Nesse contexto, o temperamento de aproximação/ abordagem tem sido definido como uma sensibilidade neurobiológica geral a estímulos positivos/desejáveis, presentes ou imaginários (como, por exemplo, uma recompensa), que provoca emoções positivas e uma predisposição comportamental aos estímulos positivos (Elliot \& Thrash, 2002; Elliot \& Thrash, 2010).

O temperamento de evitação/evasão, por sua vez, pode ser caracterizado como aquele que é instigado ou dirigido por um evento negativo/indesejável, presente ou imaginário (como, por exemplo, uma ameaça, castigo ou punição), estando orientado para evitar e prevenir o dano e a perda. Ele promove, assim, a atenção vigilante em direção ao meio ambiente, e correlacionase empiricamente com as emoções negativas (Elliot, 2008; Elliot \& Thrash, 2010).

$\mathrm{Na}$ tentativa de aprofundar o conhecimento sobre os temperamentos de aproximação e evitação, Elliot e Church (1997) propuseram o modelo hierárquico de motivação à aproximação ou evitação. De acordo com tal modelo, a meta consiste em um elemento central, em função do pressuposto de que o direcionamento para as metas é uma característica fundamental do comportamento humano, razão pela qual torna-se difícil imaginar uma ação motivada satisfatória que exclua esta característica.

A orientação para as metas se dá por meio do temperamento, que funciona como energizante do comportamento de aproximação e evitação (Elliot, 2008). O temperamento consiste, assim, em uma tendência afetiva que orienta os indivíduos em relação aos estímulos positivos ou negativos. Em outras palavras, quando os indivíduos querem alcançar uma meta, eles apresentam diferentes orientações que guiam seus comportamentos, cognições e afetos (Elliot, 2008).

O modelo hierárquico postula, ainda, a existência de objetivos, que se caracterizam por representações de objetos futuros que o indivíduo tende a abordar ou evitar. Eles consistem, portanto, no componente final do processo motivacional, e podem ser usados a serviço de diferentes temperamentos (Elliot, 2008). No modelo hierárquico, então, as metas e o temperamento a elas subjacentes encontram-se intimamente interligados, podendo ser usados para a emissão de comportamentos de aproximação (abordagem) ou de evitação (evasão) em relação a determinados objetivos.

Elliot e Thrash (2010) propuseram-se a examinar a estrutura interna de uma medida por eles desenvolvida para avaliar os temperamentos de aproximação e de evitação. Para tanto, realizaram uma série de estudos. Em um primeiro estudo, os autores buscaram avaliar diretamente os temperamentos de aproximação e evitação como dimensões de personalidade internamente consistentes e relativamente independentes, com base na suposição de que elas emergem de estruturas e processos biologicamente independentes. Nesse sentido, conduziram inicialmente uma série de estudos-piloto nos quais examinaram o conteúdo de um conjunto de itens e selecionaram os considerados mais apropriados a compor o Questionário de Temperamento de Aproximação-Evitação (Approach - Avoidance Temperament Questionnaire - ATQ), com duas dimensões (aproximação e evitação), de seis itens cada. Em seguida, o instrumento foi aplicado a uma amostra de 149 alunos de graduação (56 homens e 93 mulheres). A análise fatorial exploratória revelou 
a presença de dois fatores com consistências internas iguais a 0,80 e 0,79 , respectivamente.

Em outro estudo, os autores utilizaram a análise fatorial confirmatória para validar a estrutura de dois fatores do estudo 1. A amostra foi composta por 150 graduandos (52 do sexo masculino, 97 do sexo feminino, 1 sexo não especificado). Os resultados apontados $\left(\chi^{2}(53)=84,84, p<0,01 ; \chi^{2} / d f=1,60\right.$; $\mathrm{IFI}=0,93$; TLI $=0,91$; RMSEA $=0,63)$, bem como os índices de consistência interna ( 0,74 para aproximação e 0,81 para evitação), confirmaram a solução de dois fatores do estudo 1.

Em um terceiro estudo, os autores procuraram estabelecer a estabilidade teste-reteste das dimensões de aproximação e evitação ao longo de um período de 9 semanas. Para tanto, 161 participantes (52 homens e 109 mulheres) completaram o ATQ no tempo 1 (T1) e 9 semanas depois, no tempo 2 (T2). Os índices de ajuste $\left(\chi^{2}(42)=78,45, p<0,01 ; \chi^{2} / d f=1,87\right.$; IFI $=0,98$; $\mathrm{TLI}=0,95 ; \mathrm{RMSEA}=0,07)$ e de consistência interna (0,85 e 0,86 , respectivamente), apontaram que os resultados em T1 e T2 foram muito semelhantes ao modelo de dois fatores examinado no estudo 2 , além de indicarem a estabilidade dos temperamentos de aproximação e evitação ao longo de um período de 9 semanas.

No quarto estudo, Elliot e Thrash (2010) examinaram se o temperamento de aproximação estava relacionado à extroversão, à emotividade positiva e à sensibilidade à aproximação, e se o temperamento de evitação estava relacionado ao neuroticismo, à emotividade negativa e à sensibilidade à evasão. Compuseram a amostra 141 graduandos (32 homens, 94 mulheres, 15 com sexo não especificado). Os índices de ajuste $\left(\chi^{2}(245)=385,67, p<0,01 ; \chi^{2} / d f=1,57\right.$; $\mathrm{IFI}=0,93 ; \mathrm{TLI}=0,92 ; \mathrm{RMSEA}=0,06)$ e de consistência interna $(0,83$ e 0,87 , respectivamente) confirmaram os resultados anteriormente obtidos. Verificou-se, ainda, que o temperamento de aproximação estava associado à extroversão, à emotividade positiva e à sensibilidade à aproximação, além de que o temperamento de evitação estava associado ao neuroticismo, à emotividade negativa e à sensibilidade à evasão.

Em um quinto estudo, o propósito foi examinar as relações dos temperamentos de aproximação e de evitação com dois construtos da teoria de foco regulatório (foco de promoção crônica e foco de prevenção crônica). Participaram do estudo 139 graduandos (47 homens e 92 mulheres). Os índices de ajuste $\left(\chi^{2}(48)=92,13, \mathrm{p}<0,001 ; \chi^{2} / d f=1,92 ; \mathrm{IFI}=0,95\right.$; $\mathrm{TLI}=0,93$; RMSEA $=0,82)$ e de consistência interna $(0,78$ e 0,87 , respectivamente) também confirmaram os resultados anteriormente observados. Além disso, as correlações obtidas demonstraram que o temperamento de aproximação estava associado ao foco de promoção crônica, enquanto o temperamento de evitação associou-se à prevenção crônica.

Bipp, Kleingeld e van Dam (2015) investigaram a estrutura fatorial, de construto e preditiva do Questionário de Temperamento de Aproximação e de Evitação (ATQ) no contexto do trabalho. No primeiro estudo, os autores buscaram validar a estrutura fatorial da versão traduzida do ATQ, após os procedimentos de tradução (inglês-holandês) e retradução (holandêsinglês) destinados a garantir a equivalência de conteúdo dos itens em inglês e holandês. Participaram deste estudo 395 estudantes holandeses ( $62 \%$ do sexo masculino) de duas instituições acadêmicas diferentes. Os resultados da análise fatorial confirmatória $\left(\chi^{2} / d f=2,25\right.$; $\mathrm{TLI}=0,92 ; \mathrm{CFI}=0,94 ; \mathrm{RMSEA}=0,06)$ apontaram um bom ajuste para a solução de dois fatores, além de ter sido obtida adequada consistência interna $(0,80$ e 0,73 , aproximação e evitação, respectivamente). Tais índices mostraram-se assim semelhantes aos relatados para o questionário original (Elliot \& Thrash, 2010).

No segundo estudo, Bipp et al. (2015) investigaram a relação dos temperamentos de aproximação e evitação com o engajamento do estudante e a adaptabilidade à carreira. Os participantes foram 290 alunos holandeses de diferentes cursos universitários. Duas análises de regressão hierárquica foram conduzidas para testar as hipóteses sobre as relações dos temperamentos (aproximação e evitação) com o engajamento do estudante e a adaptabilidade à carreira. Os dados apontaram que o temperamento de aproximação estava correlacionado positivamente à adaptabilidade à carreira $(\beta=0,59, p<0,01)$ e ao engajamento do estudante $(\beta=0,27, p<0,01)$, além de que o temperamento de evitação também se correlacionou negativamente à adaptabilidade à carreira $(\beta=-0,15, p<0,01)$.

Como objetivo principal, em seu terceiro estudo, Bipp et al. (2015) buscaram fornecer mais informações sobre a validade do ATQ, verificando a validade incremental do instrumento em relação aos efeitos da orientação para as metas (posição de gestão, metas de aprendizagem, metas de abordagem de desempenho e metas de prevenção de desempenho) no engajamento no trabalho. Participaram do estudo 103 empregados holandeses de várias organizações. Os resultados apontaram a validade incremental do instrumento em relação ao engajamento no trabalho, para além da orientação para as metas.

Por fim, em seu quarto estudo, Bipp et al. (2015) objetivaram avaliar a validade do instrumento em relação ao desempenho no trabalho. Participaram do estudo 141 empregados holandeses de uma antiga 
agência governamental. As análises de regressão hierárquica apontaram que o temperamento de aproximação se correlacionou de forma positiva fraca com o desempenho na tarefa $(\beta=0,24, p<0,05)$ e o desempenho contextual $(\beta=0,22, p<0,05)$, e positivamente moderada com o desempenho criativo $(\beta=0,32, p<0,01)$.

Embora os estudos sobre a validade do ATQ venham ganhando destaque na literatura internacional (Elliot \& Thrash, 2010; Bipp et al. 2015), inexistem, até o momento, estudos destinados a buscar evidências de validade do instrumento em amostras de trabalhadores brasileiros, conforme levantamento realizado em bases de dados nacionais e internacionais (Psychinfo, Scientific Eletronic Library Online - SciELO, Portal de Periódicos Eletrônicos de Psicologia - PePSIC e Banco de Teses da CAPES), no mês de dezembro de 2017. O levantamento em tela usou os seguintes descritores: temperamento de abordagem e evasão; temperamento de aproximação e evitação; motivação de aproximação e evitação; motivação de abordagem e evasão; comportamento de aproximação e evitação; comportamento de abordagem e evasão; desempenho de aproximação e evitação; desempenho de abordagem e evasão.

Com o propósito de preencher esta lacuna, o presente estudo teve como objetivo adaptar e reunir evidências iniciais de validade de estrutura interna e de relações com variáveis externas do Questionário de Temperamento de Aproximação-Evitação, na versão adaptada por Bipp et al. (2015) ao contexto de trabalho. Tal estudo poderá contribuir para a compreensão da disposição comportamental dos indivíduos e de seu impacto sobre os comportamentos no trabalho, bem como para o desenvolvimento de práticas de recursos humanos destinadas a melhorar, dentre outros aspectos, a adaptabilidade, o engajamento e o desempenho dos trabalhadores, o que pode se repercutir em consequências positivas para suas organizações empregadoras.

$\mathrm{Na}$ análise das relações do temperamento de aproximação e evitação com variáveis externas foram adotados os construtos engajamento no trabalho, adaptabilidade à carreira e desempenho de papéis no trabalho. $\mathrm{O}$ engajamento consiste em um estado psicológico difuso, de natureza afetivo-cognitiva, que se manifesta na dedicação, vigor e absorção ao trabalho (Schaufeli, 2013). Seria assim de se esperar correlações positivas baixas entre tal construto e o temperamento de aproximação e evitação, conforme estudos realizados por Bipp et al. (2015). Nesse sentido, formulou-se a hipótese de que o temperamento de aproximação apresentaria uma correlação positiva baixa com o engajamento no trabalho (H1), enquanto o temperamento de evitação apresentaria uma correlação negativa baixa com tal constructo $(\mathrm{H} 2)$.

A adaptabilidade à carreira denota a prontidão e os recursos de um indivíduo para lidar com as tarefas atuais inerentes ao desenvolvimento de carreira e transições ocupacionais (Savickas, 2013). Ela diz respeito, portanto, à qualidade de ser apto à mudança e se ajustar a circunstâncias novas ou modificadas (Savickas, 2013). Estudos realizados por Elliot e Thrash (2010) e Bipp et al. (2015) apontaram a existência de associações positivas elevadas do temperamento de aproximação com a adaptabilidade à carreira, bem como associações negativas baixas entre o temperamento de evitação e o referido constructo. Com base em tais estudos, seria de se esperar uma associação positiva elevada do temperamento de aproximação com a adaptabilidade à carreira (H3), bem como uma associação negativa baixa entre o temperamento de evitação e o referido constructo (H4).

O desempenho no trabalho tem sido caracterizado como uma autoavaliação sobre a execução das atividades que contribuem para a resolução das questões técnicas da organização (Sonnentag \& Frese, 2002). Tal variável, de acordo com estudos de Bipp et al. (2015), costuma apresentar correlações positivas baixas com o temperamento de aproximação e negativas baixas com o temperamento de evitação. Desse modo, formulou-se a hipótese de que existiria uma correlação positiva baixa desta variável com o temperamento de aproximação (H5) e negativa baixa com o temperamento de evitação (H6).

\section{Método}

\section{Participantes}

A amostra foi composta por 453 trabalhadores brasileiros de ambos os sexos (79,2\% do sexo feminino), com profissões diversificadas e provenientes de todas as regiões do País. A idade dos participantes variou de 18 a 65 anos $(M=37,6 ; D P=9,88)$. Quanto ao estado civil, a maioria era casada $(57,2 \%)$ e não possuía filhos $(50,6 \%)$. No que tange à escolaridade, $24,1 \%$ tinham nível superior completo e $57,6 \%$ possuíam pós-graduação stricto ou lato-senso. Os participantes trabalhavam, em sua maioria, no setor privado (55\%), em cargos de nível administrativo ou operacional $(50,3 \%)$, com um tempo de experiência profissional variando de 1 a mais de 20 anos $(M=3,71 ; D P=1,59)$. Para inclusão no estudo, utilizou-se o critério de o participante estar trabalhando e possuir pelo menos um ano de experiência em sua respectiva função. 


\section{Instrumentos}

O temperamento de aproximação e evitação foi avaliado pelo Questionário de Temperamento de Aproximação e Evitação (Approach-Avoidance Temperament Questionnaire -ATQ) de Elliot e Thrash (2010), em versão adaptada ao contexto do trabalho por Bipp et al. (2015). O instrumento é composto por 12 itens, distribuídos em 2 dimensões (aproximação e evitação) de seis itens cada, a serem respondidos em uma escala tipo Likert de sete pontos, variando de 1 (Discordo fortemente) a 7 (Concordo fortemente). Exemplo de item: "Por natureza, sou uma pessoa muito nervosa". Pelo fato deste questionário não possuir uma versão para o contexto brasileiro, foi necessária a sua tradução do inglês para o português, por um especialista bilíngue. Em seguida, foi realizado o processo de retradução (backtranslation), ou seja, a tradução reversa do português para o inglês. Posteriormente, as duas versões em inglês foram comparadas por um expert. No estudo original, as dimensões de aproximação e evitação apresentaram índices de consistência interna iguais a 0,74 e 0,81 , respectivamente.

O engajamento no trabalho foi avaliado pela versão brasileira reduzida da Escala de Engajamento no Trabalho (UWES), de Schaufeli, Bakker e Salanova (2006), adaptada para o contexto brasileiro por Ferreira et al. (2016). Ela é composta por nove itens, a serem respondidos em escalas tipo Likert de sete pontos, variando de nunca (0) a sempre (6). Exemplo de item: "Sinto-me envolvido com o trabalho que realizo". A consistência interna da escala no estudo de Ferreira et al. (2016), avaliada pelo Alfa de Cronbrach, foi igual a 0,93 . No presente estudo, ela foi de 0,95.

A Adaptabilidade à carreira foi mensurada pela Escala de Adaptabilidade à Carreira (Career AdaptAbilities Scale-CAAS) de Savickas e Porfeli (2012), na versão brasileira adaptada por Teixeira, Bardagi, Lassance, Magalhães e Duarte (2012), e posteriormente revisada por Audibert e Teixeira (2015). O instrumento é composto por 24 itens, distribuídos em quatro dimensões: preocupação (6 itens), controle (6 itens), curiosidade (6 itens) e confiança (6 itens), a serem respondidos em uma escala de 5 pontos, variando de 1 (Desenvolvi pouco ou nada) a 5 (Desenvolvi extremamente bem). Exemplo de item: "Atualmente, penso sobre como será meu futuro". No estudo de Audibert e Teixeira (2015), a consistência interna das subescalas, avaliada pelo Alfa de Cronbach, foram iguais a: preocupação - 0,88 ; controle $-0,83$; curiosidade $-0,88$; e confiança $-0,89$. No presente estudo, esses índices foram iguais a: preocupação -
0,89 ; controle $-0,85$; curiosidade $-0,88$; e confiança $-0,90$.

O desempenho de papéis no trabalho foi avaliado por duas subescalas da Escala de Desempenho de Papéis de Trabalho de Griffin, Neal e Parker (2007), utilizada em amostras brasileiras por Chinelato (2016). O instrumento total é composto por vinte e sete itens, a serem respondidos em escalas tipo Likert de sete pontos, variando de 1 (nunca) a 7 (sempre). Neste estudo, porém, foram utilizados somente seis itens, correspondentes às subescalas de desempenho individual na tarefa (3 itens) e desempenho de equipe (3 itens), assim como no estudo de Chinelato (2016). Exemplo de item: "Realizei bem as partes principais do meu trabalho no último mês". No estudo de Chinelato (2016), as escalas apresentaram índices de 0,82 e 0,88 , respectivamente, enquanto que, no presente estudo, eles foram de 0,91, para o desempenho individual, e de 0,87 , para o desempenho de equipe.

\section{Procedimentos de coleta e análise de dados}

A pesquisa foi submetida ao Comitê de Ética em Pesquisa da instituição dos autores e aprovada com o CAAE no 78193117.9.0000.5289. Após sua aprovação, a coleta de dados foi realizada totalmente em versão online, a partir da ferramenta Google Docs. Os convites à participação foram enviados diretamente por e-mail, além de serem postados em redes sociais como Facebook, Instagram e Whatsapp. Vale ressaltar que na aplicação houve concordância prévia sobre a participação na pesquisa, mediante o preenchimento do Termo de Consentimento Livre e Esclarecido (TCLE), que constava como parte inicial do questionário.

As análises fatoriais confirmatórias foram realizadas por meio do software MPlus (Muthén \& Muthén, 2010), versão 6.12, com a utilização do estimador WLSMV em todos os modelos testados. Os índices de ajuste avaliados e os valores de referência adotados foram: $\chi^{2} / \mathrm{gl}<5$; CFI $>0,95$; TLI $>0,95$; RMSEA $<0,05$ (Hu \& Bentler, 1995). A invariância referente ao sexo e à escolaridade dos participantes foi testada por meio da análise de invariância multigrupo, com auxílio do software Mplus. Foram avaliadas a invariância configural (M1), métrica (M2), escalar (M3) e residual (M4). Para tanto, foram analisados os aumentos no $\triangle \mathrm{CFI}$, com valores inferiores a 0,01 sendo considerados como invariantes (Damásio, 2013).

A avaliação da consistência interna foi realizada por meio do coeficiente Alfa de Cronbach. A investigação das correlações com variáveis externas do Questionário de Temperamento de Aproximação e Evitação (ATQ) foi efetuada por meio do cálculo de correlações de Spearman, em virtude dos dados terem 
sido considerados como categóricos. $\mathrm{Na}$ interpretação da magnitude dos coeficientes de correlação foram adotados os critérios de Miles e Shevlin (2001), que os classificam de acordo com os seguintes intervalos: 0,10 - 0,29 (baixa); 0,30 - 0,49 (moderada); e, maior que 0,50 (elevada).

\section{Resultados}

Para verificar a validade de estrutura interna da escala foram testados quatro diferentes modelos, que apresentaram os seguintes índices de ajuste: modelo unifatorial $\left(\chi^{2}(\mathrm{gl})=1453,567\right.$ (54); TLI $=0,293$; $\mathrm{CFI}=0,421$; $\mathrm{RMSEA}=0,239)$; modelo de dois fatores correlacionados $\left(\chi^{2}(\mathrm{gl})=314.406\right.$ (53); TLI $=0,865$; $\mathrm{CFI}=0,892$; RMSEA $=0,104)$; modelo de dois fatores não correlacionados $\left(\chi^{2}(\mathrm{gl})=207,204(54) ; \mathrm{TLI}=0,923\right.$; $\mathrm{CFI}=0,937$; $\mathrm{RMSEA}=0,079)$; modelo ajustado de dois fatores não correlacionados $\left(\chi^{2}(\mathrm{gl})=168,657\right.$ (52); TLI $=0,939 ; \mathrm{CFI}=0,952 ; \mathrm{RMSEA}=0,070)$. Tais modelos, à exceção do unifatorial, apresentaram bons índices de ajuste, com pequenas diferenças em termos estatísticos. Ainda assim, porém, o modelo ajustado de dois fatores não correlacionados foi o que obteve os melhores índices de ajuste e foi, desse modo, escolhido para representar a estrutura interna do instrumento.

Os resultados demonstraram, portanto, que a versão brasileira do questionário de Temperamento de Aproximação e Evitação permaneceu com os 12 itens da versão original, distribuídos em duas dimensões, com ajustes relacionados à correlação entre os erros dos itens 9 e 10 e 11 e 12 . A análise de invariância multigrupo por sexo indicou que, para os quatro modelos testados (invariância configural $(\mathrm{M} 1)=0,908$; métrica $(M 2)=0,908$; escalar $(M 3)=0,899$; residual $(\mathrm{M} 4)=0,894)$ não houve variações do CFI superiores a 0,01. De modo semelhante, na análise de invariância multigrupo por escolaridade $(\mathrm{M} 1=0,859 ; \mathrm{M} 2=0,850$; $\mathrm{M} 3=0,853 ; \mathrm{M} 4=0,845)$, não houve, também, variações do CFI superiores a 0,01 .

Na Tabela 1, são apresentados os parâmetros padronizados encontrados, sendo possível observar que, no intervalo de confiança de $95 \%$, não está incluído nenhum valor 0 , além de que todos os valores de razão crítica são maiores que 1,96, em uma indicação de que os parâmetros avaliados são significativamente diferentes de 0 e podem, consequentemente, ser considerados úteis ao modelo. As cargas fatorais de todos os itens foram altas (acima de 0,50), à exceção do item 9 , que apresentou carga fatorial de 0,48 . Os índices de consistência interna do questionário, calculados por meio do Alfa de Cronbach, foram iguais a 0,79, para a dimensão de temperamento de aproximação, e de 0,74 , para o temperamento de evitação, indicando, assim, consistência interna adequada de ambas as dimensões.

A Tabela 2 apresenta as médias, os desviospadrão e as correlações entre as variáveis do estudo. O temperamento de aproximação apresentou correlação positiva baixa $(\rho=0,24 ; p<0,01)$ com o engajamento no trabalho, enquanto o temperamento de evitação apresentou correlação negativa moderada $(\rho=-0,33 ; p<0,01)$ com o referido construto, o que confirmou totalmente a hipótese 1 e, parcialmente, a hipótese 2. No que tange à correlação do ATQ com a

TABELA 1

Cargas fatoriais dos itens do Questionário de Temperamento de Aproximação e Evitação

\begin{tabular}{llccc}
\hline Itens & Cargas & Erros & Razão Crítica & $\begin{array}{c}\text { Intervalo de } \\
\text { Confiança }\end{array}$ \\
\hline $\begin{array}{l}\text { Aproximação } \\
\text { Temp2 }\end{array}$ & 0,66 & 0,03 & 20.09 & 0,60 a 0,73 \\
Temp4 & 0,78 & 0,03 & 27.90 & 0,72 a 0,84 \\
Temp5 & 0,61 & 0,03 & 19.06 & 0,54 a 0,67 \\
Temp8 & 0,71 & 0,03 & 26.43 & 0,66 a 0,77 \\
Temp10 & 0,53 & 0,04 & 14.33 & 0,46 a 0,60 \\
Temp11 & 0,78 & 0,03 & 29.02 & 0,73 a 0,83 \\
Evitação & & & 0,42 a 0,59 \\
Temp1 & 0,50 & 0,04 & 12.04 & 0,44 a 0,59 \\
Temp3 & 0,04 & 13.43 & 0,78 a 0,89 \\
Temp6 & 0,52 & 32.27 & 0,56 a 0,69 \\
Temp7 & 0,83 & 19.01 & 0,41 a 0,55 \\
Temp9 & 0,63 & 0,03 & 12.60 & 0,62 a 0,74 \\
Temp12 & 0,48 & 0,04 & 22.32 & \\
\hline
\end{tabular}

Temp $=$ Temperamento 
TABELA 2

Médias, desvios - padrão e correlações entre as variáveis do estudo

\begin{tabular}{|c|c|c|c|c|c|c|c|c|c|c|}
\hline & $M D$ & $D P$ & 1 & 2 & 3 & 4 & 5 & 6 & 7 & 8 \\
\hline 1. T evitação & 3,82 & 1,33 & - & & & & & & & \\
\hline 2. T aproximação & 5,61 & 1,06 & $-0,37$ & - & & & & & & \\
\hline 3. Adap. preocupação & 3,47 & 0,87 & $-0,20$ & 0,28 & - & & & & & \\
\hline 4. Adap. controle & 3,88 & 0,78 & $-0,26$ & 0,28 & 0,57 & - & & & & \\
\hline 5. Adap. curiosidade & 3,63 & 0,82 & $-0,23$ & 0,28 & 0,58 & 0,70 & - & & & \\
\hline 6. Adap. confiança & 3,98 & 0,78 & $-0,21$ & 0,26 & 0,48 & 0,67 & 0,69 & - & & \\
\hline 7. Engaj. Trabalho & 3,45 & 1,48 & $-0,33$ & 0,24 & 0,34 & 0,39 & 0,39 & 0,39 & - & \\
\hline 8. Des. Individual & 5,31 & 1,32 & $-0,19$ & 0,22 & 0,26 & 0,38 & 0,38 & 0,45 & 0,47 & - \\
\hline 9. Des. Equipe & 5,30 & 1,31 & $-0,15$ & 0,19 & 0,25 & 0,41 & 0,33 & 0,43 & 0,43 & 0,79 \\
\hline
\end{tabular}

Notas: Todas as correlações foram significativas ao nível de $p<0,01(\mathrm{n}=453)$.

variável adaptabilidade à carreira, observou-se que o temperamento de aproximação apresentou correlações positivas baixas com todas as quatro dimensões do construto (preocupação $-\rho=0,28 ; p<0,01$; controle $-\rho=0,28 ; p<0,01$; curiosidade $-\rho=0,28 ; p<0,01$; confiança $-\rho=0,26 ; p<0,01)$, o mesmo ocorrendo nas correlações com o temperamento de evitação, que foram negativas baixas (preocupação $-\rho=-0,20$; $p<0,01)$; controle $-\rho=-0,26 ; p<0,01$; curiosidade $-\rho=-0,23 ; p<0,01$; confiança $-\rho=-0,21 ; p<0,01)$. Com isso, as hipóteses 3 e 4 também foram totalmente confirmadas.

Com a escala de desempenho de papéis no trabalho, os dados apontaram uma correlação positiva baixa entre o temperamento de aproximação e o desempenho individual $(r \rho=0,22 ; p<0,01)$ e o desempenho de equipe $(\rho=0,19 ; p<0,01)$, bem como correlações negativas baixas do temperamento de evitação com o desempenho individual $(\rho=-0,19 ; p<0,01)$ e o desempenho de equipe $(\rho=-0,15 ; p<0,01)$. Logo, as hipóteses 5 e 6 também se confirmaram totalmente.

\section{Discussão}

O presente trabalho teve como objetivo adaptar e reunir evidências iniciais de validade de estrutura interna e de relações com variáveis externas do Questionário de Temperamento de Aproximação-Evitação, na versão adaptada ao contexto de trabalho por Bipp et al. (2015), em uma amostra de trabalhadores brasileiros. Após a análise fatorial confirmatória, observou-se que o modelo de melhor ajuste foi o de dois fatores não correlacionados, com ajustes entre dois pares de erros. Tais resultados confirmam o modelo original do questionário de temperamento de aproximação e evitação de Elliot e Thrash (2010), visto que, no estudo original, bem como no de Bipp et al. (2015), também foram encontrados modelos de dois fatores não correlacionados, em uma indicação de que a estrutura do instrumento vem se mantendo, independentemente da diversidade de padrões culturais existentes entre as sociedades norte-americana, holandesa e brasileira.

A amostra apresentou dados heterogêneos quanto ao sexo e a escolaridade, sendo majoritariamente representada por homens com ensino superior completo e pós-graduação. No entanto, a análise da invariância multigrupos por sexo e por escolaridade indicou a estabilidade da estrutura do questionário para os diferentes grupos formados em relação a essas variáveis. Tais resultados sugerem, portanto, que há equivalência de respostas entre os indivíduos desses grupos apesar da variabilidade da amostra. Em outras palavras, as médias obtidas nos diferentes grupos não se encontram enviesadas (Damásio, 2013), em uma indicação de que tais variáveis demográficas provavelmente não atuam como moderadoras do modelo proposto.

Os índices de consistência interna do questionário, calculados por meio do Alfa de Cronbach, foram satisfatórios para as dimensões de temperamento de aproximação $(\alpha=0,79)$ e de temperamento de evitação $(\alpha=0,75)$, e se assemelham aos de Elliot e Thrash (2010), que obtiveram índices variando de 0,74 a 0,85 , no temperamento de aproximação, e de 0,79 a 0,87 , no temperamento de evitação. Eles se mostraram também congruentes com o estudo de Bipp et al. (2015), que obteve índices de 0,80 e 0,73 , respectivamente. Tais estudos apontam, portanto, que o instrumento vem apresentando precisão adequada nas estimativas dos escores de temperamento de aproximação e evitação.

No que diz respeito às correlações do ATQ com variáveis externas, foi possível verificar a existência de uma correlação positiva baixa do temperamento de aproximação com o engajamento no trabalho, e 
negativa moderada desta variável com o temperamento de evitação. Tais resultados convergem com estudos anteriores, como o de Bipp et al. (2015), que também observaram correlações dessa natureza entre tais construtos. Considerando-se, ainda, que o neuroticismo é um traço característico do temperamento de evitação, os achados ora obtidos também convergem com o estudo de Langelaan, Bakker, Van Doornen e Schaufeli (2006), no qual foi encontrada uma correlação negativa entre o neuroticismo e o engajamento no trabalho. Uma possível explicação para tal resultado é a de que o temperamento de aproximação pode ser visto como motor do engajamento (Langelaan et al., 2006), isto é, como um aspecto individual que leva os empregados a se tornarem mais envolvidos e entusiasmados com seu trabalho (Bakker, 2014). A predisposição à evitação e à sensibilidade à punições, por sua vez, leva os indivíduos a se concentrarem em aspectos negativos de seu trabalho, o que justifica sua falta de engajamento no trabalho (Bipp et al. 2015).

No que diz respeito à adaptabilidade à carreira, o temperamento de aproximação correlacionou-se de forma positiva baixa com as dimensões dessa variável, enquanto o temperamento de evitação apresentou correlações negativas baixas com as mesmas. Tais resultados assemelham-se parcialmente aos estudos de Bipp et al. (2015), já que esses autores obtiveram correlações positivas altas entre a dimensão geral de adaptabilidade à carreira e o temperamento de aproximação, além de correlações negativas baixas com o temperamento de evitação. Acrescente-se a isso o fato de a extroversão constituir-se em um componente psicológico relacionado ao temperamento de aproximação e, ao mesmo tempo, em um indicador de traços de adaptabilidade à carreira (Savickas \& Porfeli, 2012), o que também corrobora o presente achado. Tais resultados podem ser explicados pelo fato de que os indivíduos com tendências comportamentais para a aproximação costumam dirigir seus esforços para os estímulos positivos, o que lhes leva a buscar em maior grau o sucesso profissional, o desenvolvimento pessoal e a competência intrapessoal (Bipp \& Demerouti, 2015; Bipp et al., 2015; Elliot \& Thrash, 2002), razão pela qual adaptam-se mais facilmente a sua carreira. Outrossim, os indivíduos com forte temperamento de evasão têm maiores dificuldades de lidar com as demandas do trabalho, o que dificulta sua adaptação às mudanças ocorridas em suas carreiras (Bipp et al. 2015; Bipp \& Demerouti, 2014).

$\mathrm{Na}$ análise das relações do ATQ com o desempenho de papéis no trabalho, verificou-se que o temperamento de aproximação apresentou uma correlação positiva baixa com as dimensões de desempenho individual e de desempenho de equipe, sendo que o temperamento de evasão apresentou correlações negativas baixas com o desempenho individual e o desempenho de equipe. Tais resultados mostram-se congruentes com os observados por Bipp et al. (2015), que também encontraram correlações baixas do temperamento de aproximação com o desempenho na tarefa e o desempenho criativo, e por Ferris et al. (2011), que verificaram que o temperamento de evasão tinha efeitos negativos sobre o desempenho da tarefa e o desempenho criativo. Constatou-se, assim, que indivíduos motivados à abordagem tendem a apresentar melhor desempenho nas tarefas, sendo que os indivíduos orientados para a evitação tendem a apresentar pior desempenho em suas tarefas e funções (Ferris et al. 2011).

Merece destaque, ainda, o fato de a maioria das correlações com as variáveis externas terem sido baixas, o que pode se dever ao fato de os traços de temperamento não serem muito suscetíveis a mudanças (Strelau, 1998). Dito de outra forma, os temperamentos de aproximação e evitação consistem em características estáveis de personalidade, enquanto as variáveis externas com as quais eles foram correlacionados são mais instáveis, em função de sofrerem mais influências dos contextos aos quais os indivíduos estão expostos, o que justificaria as baixas correlações ora obtidas.

No que tange às limitações do presente estudo, salienta-se o uso de instrumentos de autorrelato, que podem afetar os resultados, em virtude de os indivíduos tenderem a responder com alta desejabilidade social. Além disso, como o estudo foi de corte transversal, os achados obtidos não permitem a realização de inferências de causalidade nas relações encontradas. Em relação a uma agenda futura de pesquisas, seria interessante que estudos futuros procurassem investigar se a estrutura de dois fatores do ATQ permanece invariante em outras culturas e em grupos ocupacionais específicos. Outros estudos poderiam ainda verificar as correlações dos temperamentos de aproximação e de evitação com outros recursos de personalidade característicos das situações laborais (como a personalidade empreendedora e a personalidade proativa) e com os estilos de liderança.

De todo modo, é possível concluir que o Questionário de Temperamento de AproximaçãoEvitação apresentou evidências iniciais de validade de estrutura interna e de relações com outras variáveis em uma amostra de trabalhadores brasileiros. Tais evidências recomendam seu uso em investigações futuras destinadas à análise e compreensão do impacto dos comportamentos de temperamento de aproximação e de evitação em atitudes e comportamentos emitidos no contexto de trabalho. 


\section{Referências}

Arafat, S. M. Y. (2016). Cross cultural adaptation \& psychometric validation of instruments: Step-wise description. International Journal of Psychiatry, 1(1), 1-4. (DOI INEXISTENTE)

Audibert, A. \& Teixeira, M. A. P. (2015). Escala de Adaptabilidade de Carreira: Evidências de validade em universitários brasileiros. Revista Brasileira de Orientação Profissional, 16(1), 83-93. (DOI INEXISTENTE)

Bakker, A. B. (2014). Daily fluctuations in work engagement: An overview and current directions. European Psychologist, 19(4), 227-236. https://doi.org/10.1027/1016-9040/a000160

Bipp, T., Kleingeld, P. A. M., \& van Dam, K. (2015). Approach and avoidance temperament: An examination of its construct and predictive validity at work. European Journal of Psychological Assessment, 33(3), 196-206. https://doi.org/10.1027/1015-5759/a000285

Bipp, T. \& Demerouti, A. (2015). Which employees craft their jobs and how? Basic dimensions of personality and employees' job crafting behavior. Journal of Occupational and Organizational Psychology, 88(4), 631-655. https://doi.org/10.1111/joop.12089

Chinelato, R. S. C. (2016). Expandindo a rede nomológica do engajamento no trabalho em amostras brasileiras (Tese de Doutorado). Programa de Pós-Graduação em Psicologia, Universidade Salgado de Oliveira. Niterói. (DOI INEXISTENTE)

Damásio, B. F. (2013). Contribuições da análise fatorial confirmatória multigrupo (AFCMG) na avaliação de invariância de instrumentos psicométricos. Psico-USF, 18(2), 211-220. https://doi.org/10.1590/S141382712013000200005

Elliot, A. J. (2008). Approach and avoidance motivation. In A. Elliot (Ed.), Handbook of approach and avoidance motivation (pp. 3-14). New York, NY: Taylor \& Francis. (DOI INEXISTENTE)

Elliot, A. J. \& Church, M. A. (1997). A hierarchical model of approach and avoidance achievement motivation. Journal of Personality and Social Psychology, 72(1), 218-232. https://doi.org/10.1037/0022-3514.72.1.218

Elliot, A. J. \& Thrash, T. M. (2002). Approach-avoidance motivation in personality: Approach and avoidance temperaments and goals. Journal of Personality and Social Psychology, 82(5), 804-818. https://doi. org/10.1037//0022-3514.82.5.804

Elliot, A. J. \& Thrash, T. M. (2010). Approach and avoidance temperament as basic dimensions of personality. Journal of Personality, 78(3), 865-906. https://doi.org/10.1111/j.1467-6494.2010.00636.x

Ferris, D. L., Rosen, C. R., Johnson, R. E., Brown, D. J., Risavy, S. D., \& Heller, D. (2011). Approach or avoidance (or both?): Integrating core self-evaluations within an approach/avoidance framework. Personnel Psychology, 64(1), 137-161. https://doi.org/10.1111/j.1744-6570.2010.01204.x

Ferreira, M. C., Valentini, F., Damásio, B. F., Mourão, L., Porto, J. B., Chinelato, R. S. C., Novaes, V. P., \& Pereira, M. M. (2016). Evidências adicionais de validade da UWES-9 em amostras brasileiras. Estudos de Psicologia, 21(4), 435-445. https://doi.org/10.5935/1678-4669.20160042

Griffin, M. A., Neal, A., \& Parker, S. K. (2007). A new model of work role performance: Positive behavior in uncertain and interdependent contexts. Academy of Management Journal, 50(2), 327-347. https://doi.org/10.5465/ amj.2007.24634438

Hu, L. T. \& Bentler, P. (1995). Evaluating model fit. In R. H. Hoyle (Ed.), Structural equation modeling. Concepts, issues, and applications (pp. 76-99). London: Sage. (DOI INEXISTENTE)

Langelaan, S., Bakker, A. B., Van Doornen, L. J. P., \& Schaufeli, W. B. (2006). Burnout and work engagement: Do individual differences make a difference? Personality and Individual Differences, 40(3), 521-532. https://doi. org/10.1016/j.paid.2005.07.009

Miles, J. N. V. \& Shevlin, M. E. (2001). Applying regression and correlation: A guide for students and researchers. London: Sage. (DOI INEXISTENTE)

Muthén, L. K. \& Muthén, B. O. (2010). Mplus user's guide (6th ed.). Los Angeles, CA: Muthén \& Muthén. (DOI INEXISTENTE)

Savickas, M. L. (2013). The theory and practice of career construction. In S. D. Brown \& R. W. Lent (Eds.), Career development and counselling: Putting theory and research to work (2nd ed., pp. 147-183). Hoboken: Wiley. (DOI INEXISTENTE) 
Savickas, M. L. \& Porfeli, E. J. (2012). Career Adapt-Abilities Scale: Construction, reliability, and measurement equivalence across 13 countries. Journal of Vocational Behavior, 80(3), 661-673. https://doi.org/10.1016/j. jvb.2012.01.011

Schaufeli, W. B. (2013). What is engagement? In C. Truss, K. Alfes, R. Delbridge, A. Shantz, \& E. Soane (Eds.), Employee engagement in theory and practice (pp. 293-320). London: Routledge. (DOI INEXISTENTE)

Schaufeli, W. B., Bakker, A. B., \& Salanova, M. (2006). The measurement of work engagement with a short questionnaire: A cross-national study. Educational and Psychological Measurement, 66(4), 701-716. https://doi. org/10.1177/0013164405282471

Sonnentag, S. \& Frese, M. (2002). Performance concepts and performance theory. In S. Sonnentag (Ed.), Psychological management of individual performance (pp. 3-27). London: John Wiley \& Sons. https://doi. org/10.1002/0470013419

Strelau, J. (1998). Temperament: A psychological perspective. New York, NY: Plenum. (DOI INEXISTENTE)

Teixeira, M. A. P., Bardagi, M. P., Lassance, M. C. P., Magalhães, M. O., \& Duarte, M. E. (2012). Career adaptabilities scale - Brazilian form: Psychometric properties and relationships to personality. Journal of Vocational Behavior, 80(3), 680-685. https://doi.org/10.1016/j.jvb.2012.01.007

Tooby, J. \& Cosmides, L. (1990). The past explains the present: Emotional adaptations and the structure of ancestral environments. Ethology and Sociobiology, 11(4-5), 375-424. https://doi.org/10.1016/0162-3095(90)90017-Z

\footnotetext{
Dados das autoras:

Especificação de autoria:

Endereço para correspondência:

Alexandra de Oliveira Rodrigues Marçulo

Programa de Pós-Graduação em Psicologia da UNIVERSO

Rua Marechal Deodoro, 217- Bloco A - 2을 andar - Centro

24030-060, Niterói, RJ, Brasil

E-mail: alexandra.psique@gmail.com

Recebido em: 27/12/2017.

Aceito em: 16/01/2019.

Publicado em: 05/08/2019.
}

Alexandra de Oliveira Rodrigues Marçulo - Mestre, Universidade Salgado de Oliveira.

Maria Cristina Ferreira - Doutora, Universidade Salgado de Oliveira. mcris@centroin.com.br

Michelle Morelo Pereira - Mestre, Universidade Salgado de Oliveira.mi_morelo@hotmail.com

Todos os autores contribuíram igualmente para produção da pesquisa e do manuscrito. 